\title{
Levels of insulin related growth factor 1 in osteoarthritis of the knee
}

\author{
T E McAlindon, J D Teale, P A Dieppe
}

\begin{abstract}
Objectives Insulin related growth factor 1 (IGF1) is an important growth promoting peptide which has anabolic effects on in vitro cartilage metabolism. The fact that serum levels tend to decrease in elderly subjects while osteoarthritis (OA) increases in prevalence suggests that suboptimal levels may play a part in the development of OA. This study therefore considers the hypothesis that IGF1 levels are low in subjects with OA of the knee.

Methods Serum IGF1 levels in a community derived sample with symptomatic knee OA were compared with those in normal controls matched by age and gender. Overall radiographic score, osteophyte score, sclerosis score, and joint space narrowing score were correlated with IFG1 levels.
\end{abstract}

Results One hundred and seventyseven elderly subjects ( 83 with knee pain, 94 without knee pain) participated, allowing the identification of 78 casecontrol pairs matched by age and gender (mean $71.8 v 71.9$ years, SD 7.6 for the two groups). No difference was found in serum IGF1 levels between the cases and controls (mean(SD) $15 \cdot 9(4 \cdot 7) \mathrm{nmol} / \mathrm{l}$, for cases, $16 \cdot 4(5 \cdot 4)$ for controls). Significant negative correlations were found between IGF1 levels and age, total radiographic score, osteophyte score, and sclerosis score. In a multiple regression model, however, only age remained significant.

Conclusions This study provides no evidence that serum IGF1 levels are low in OA. Apparent correlations with radiographic features of OA may be due to the confounding effects of age.

(Ann Rheum Dis 1993; 52: 229-231)

Osteoarthritis (OA) is a common painful and disabling disease, the prevalence of which increases rapidly with age such that pathological features of the disorder are almost universal in certain joints by the time of death. ${ }^{1-3}$ The hallmark of OA is focal cartilage loss, thought to result from the disruption of the balance between cartilage synthesis and degradation regulated by chondrocytes under the influence of mechanical and humoral factors. ${ }^{4}$ Insulin related growth factor 1 (IGF1) appears to play an important part in this homeostasis, and addition of IGF1 to chondrocyte culture systems results in dose dependent increases in proteoglycan and type II collagen synthesis. ${ }^{4}$ These facts, together with the knowledge that serum concentrations decrease substantially with age, suggest that suboptimal IGF1 production may play a part in the generation of OA. Strength is added to this hypothesis by the demonstration of low serum IGF1 levels in patients with OA compared with controls. ${ }^{6}$ We have therefore sought to confirm this finding and to investigate possible differential effects of serum IGF1 levels in terms of cartilage loss and bony growth

\section{Subjects and methods}

SUBJECTS

These were recruited from a community survey of OA of the knee in Bristol which has been reported previously. ${ }^{7}$ Following this 179 subjects with knee pain and 179 without knee pain matched by age and gender were contacted by telephone and agreed to attend the Bristol Royal Infirmary for knee radiographs and clinical assessment. The first 200 were asked if they would consent to a blood test.

Cases were required to have symptomatic radiographic OA of the knee with definite joint space narrowing in at least one compartment of one or both knees (equivalent to Kellgren and Lawrence grade $\geqslant 2$ ). Controls were matched by gender, race, year of birth, and were required to have normal knee radiographs.

\section{KNEE PAIN}

The question about knee pain was taken verbatim from the Health and Nutrition Examination Survey, ${ }^{8}$ which asks: (a) have you ever had pain in or around a knee on most days for at least a month? and (b) if so, have you had any knee pain during the last year?

An affirmative response to the two parts of the question was required for a subject to be designated 'knee pain positive'. For the purposes of analysis a score was derived from this in which $0=$ no pain, $1=$ pain in one knee, and $2=$ pain in both knees.

\section{RADIOGRAPHS}

Weightbearing anteroposterior and recumbent lateral knee radiographs were taken using a standardised technique. The three compartments of each knee were scored by a single observer (TEM) for joint space narrowing 
(0-2), osteophytosis (0-2), and sclerosis $(0,1)$ using the principles of Kellgren and Lawrence. ${ }^{9}$ These were than summated to form overall scores reflecting each feature (joint space narrowing and osteophytosis, $0-12$; sclerosis, $0-6)$ as well as overall knee OA $(0-30)$. The repeatability of this system was investigated using 100 knee radiographs scored twice in a blinded fashion and was shown to be good (correlation coefficient $0.69 ; \mathrm{p}<0.001$ )

INSULIN RELATED GROWTH FACTOR 1

Blood samples were centrifuged within three hours of venesection and serum samples were frozen and stored at $-70^{\circ} \mathrm{C}$. Levels of $\mathrm{IGF} 1$ were measured using a conventional double antibody radioimmunoassay following initial acid/ethanol extraction to minimise the effects of binding proteins. Details of the performance of this assay and its between batch variability, sensitivity, and specificity have been reported previously. ${ }^{10}$

\section{STATISTICAL ANALYSIS}

Differences between cases and controls were investigated using Student's paired $t$ test and relations between variables were investigated in the combined subsamples using linear regression and Pearson correlation coefficients. The independent contributions of age, gender, and radiographic features to the explained variance in the IGF1 levels were explored using multiple linear regression. In this model age, gender, osteophyte score, joint space narrowing score, and sclerosis score were used as dependent variables with the IGF1 level as the independent variable.

\section{Results}

SUBJECTS

Serum samples and complete data were available from 177 subjects ( 83 with knee pain, 94 without knee pain) recruited from the community. The age distributions were similar between these subsamples $\left(\chi^{2}=2 \cdot 9 ; \mathrm{p}=0 \cdot 41\right)$. Radiographic evaluation of this group allowed the identification of 78 case-control pairs ( 31 male, 47 female). The mean ages were 71.8 years for cases, 71.9 years for controls, with standard deviations of 7.6 years for the two groups.

RADIOGRAPHIC SCORES

In the group with OA these were approximately normally distributed about a mean(SD) of $8 \cdot 2(3 \cdot 1)$ (median 8 ) (see table 1).

Table 1 Distribution of severity of radiographic knee osteoarthritis in the patients (cases)

\begin{tabular}{ll}
\hline Radiographic score & No (\%) of patients \\
\hline $1-5$ & $17(22)$ \\
$6-10$ & $41(53)$ \\
$11-15$ & $20(26)$ \\
$>15$ & $0(0)$ \\
\hline
\end{tabular}

Table 2 Pearson's correlation coefficients for serum insulin related growth factor 1 levels with knee pain and with radiographic variables

\begin{tabular}{llc}
\hline & Correlation & $p$ Value \\
\hline Age & -0.31 & $<0.001$ \\
Total radiographic score & -0.17 & 0.027 \\
Osteophyte score & -0.20 & 0.009 \\
Sclerosis score & -0.14 & 0.070 \\
Joint space score & -0.08 & NS \\
Knee pain & -0.12 & NS \\
\hline
\end{tabular}

INSULIN RELATED GROWTH FACTOR 1 LEVELS Mean IGF1 levels were identical for the case and control groups (mean(SD) 15.9(4.7) $\mathrm{nmol} / \mathrm{l}$ for cases, $16 \cdot 4(5 \cdot 4) \mathrm{nmol} / 1$ for controls) and no difference was found between paired cases and controls (paired $t=0.71 ; \mathrm{p}=0.48$; degrees of freedom 77).

CORRELATIONS

In the whole sample IGF1 levels correlated most strongly with age (table 2). Significant correlations were also found with osteophytosis and sclerosis scores and with the overall knee OA score.

\section{MULTIPLE LINEAR REGRESSION}

Ten per cent $\left(r^{2}=0 \cdot 102\right)$ of the overall variance in IGF1 levels were explained by the model. Of the dependent variables (age, gender, osteophyte score, joint space narrowing score, and sclerosis score) only age was found to make a significant contribution $(62 \cdot 7 \%, \mathrm{p}=0 \cdot 0006)$.

\section{Discussion}

This study shows that serum IGF1 levels are normal in community derived subjects with symptomatic knee OA when carefully controlled by age, and provides no evidence associating low levels with cartilage loss or high levels with bone growth.

Certain factors need to be taken into account when interpreting this negative result. The study sample, though contacted in random order, cannot be assumed to be representative of the population and it is possible that biases in favour of healthier and fitter individuals attending for assessment were operative. This might be important if other variables such as muscle strength influenced IGF1 levels, though such an association was not found in this sample.

Comorbidity was also not taken into account in this study but would be expected to enhance rather than obscure any apparent difference in IGF1 levels between cases and controls.

More important is the point is that our observations are based around subjects with knee OA. The results may therefore not necessarily extrapolate to other forms of $\mathrm{OA}$ and it is possible that variations in study populations may account for the differences observed with previous work in this field. ${ }^{6}$ Moreover, the fact that OA at sites other than the knee joint was not excluded in the control group could potentially obscure low IGF1 levels in OA. The fact, however, that IGF1 
levels in each group were almost identical, and the tendency of knee OA to associate with the disorder at other sites would mitigate against a false negative resulting from our methodology. ${ }^{11}$

We use joint space narrowing as a surrogate for cartilage loss and osteophytosis for bony growth. It is conceivable that these were inappropriate choices and comparison of radiographic joint space narrowing with arthroscopic evidence of cartilage damage has shown significant discrepancies. ${ }^{12}$ At present, however, no better measure exists which is suitable for use in community based studies.

The most likely explanation of our results is that serum IGF1 levels do not reflect the local tissue environment. The discovery of carrier proteins which influence IGF1 activity and the fact that other cytokines (transforming growth factor $\beta$, interleukin 1) are likely to participate in cartilage homeostasis also needs to be taken into account. ${ }^{4} 10$

In conclusion, this study shows negative correlations between features of radiographic $\mathrm{OA}$ and serum IGF1 levels but suggests that these may be due to the confounding effects of age. Further work in this field should ensure meticulous control for age and tight disease classification.
This work was supported by an Arthritis and Rheumatism Junior Research Fellowship.

1 Van Saase J L C M, Van Romunde L K J, Cats A, Vandenbroucke J P, Valkenberg H A. Epidemiology of osteoarthritis: Zoetermeer survey. Comparison of radiological osteoarthritis in a Dutch population with that in 10 other populations. Ann Rheum Dis 1989; 48 271-80.

2 Badley E M, Thompson R P, Wood P H N. The prevalence and severity of major disabling conditions. Int $\mathcal{f}$ Epidemio 1978; 7 : 145-51.

3 Heine J. Über die arthritis deformans. Virch Arch 1926; 260: 521-663.

4 Morales T I, Hascall V C. Factors involved in the regulation of proteoglycan metabolism in articular cartilage. Arthritis Rheum 1989; 32: 1197-201.

5 Franchimont P, Bassleer C, Henrotin Y. Effects of drugs and hormones on cartilage repair. $\mathcal{F}$ Rheumatol 1989; 16 (suppl 18): 5-9.

6 Moskowitz R W, Boja B, Denko C W. The role of growth factors in degenerative joint disorders. $\mathcal{F}$ Rheumatol 1991 18 (suppl 27): 147-8.

7 McAlindon T E, Cooper C, Kirwan J R, Dieppe P A. Knee pain and disability in the community. $B r \mathcal{F}$ Rheumatol 1992; 31: 189-92.

8 Basic data on arthritis of the hip, knee and sacro-iliac joints in adults ages 25-74, United States, 1971-1975. National in adults ages 25-74, United States, 1971-1975. Nation
Center for Health Statistics series 11, No 213, 1979.

9 Kellgren J H, Lawrence J S. The epidemiology of chronic rheumatism: atlas of standard radiographs of arthritis. Oxford: Blackwell Scientific, 1963.

10 Teale J D, Marks V. Inappropriately elevated plasma insulin-like growth factor II in relation to suppressed insulin-like growth factor I in the diagnosis of non-islet cell tumour hypoglycaemia. Clin Endocrinol 1990; 33: 87-98.

11 Lawrence J S. Rheumatism in populations. London: Heinemann, 1977.

12 Fife R S, Brandt K D, Braunstein E M, et al. Relationship between arthroscopic evidence of cartilage damage and radiographic evidence of joint space narrowing in early osteoarthritis of the knee. Arthritis Rheum 1991; 34: 377-82. 